(1)

George Fox

UNIVERSITY
Digital Commons @ George Fox University

Faculty Publications - School of Physical

Therapy

School of Physical Therapy

2013

Clinical Outcomes and Static and Dynamic Assessment of Foot

Posture After Lateral Column Lengthening Procedure

Heather Barske

Ruth Chimenti

Josh Tome

Elizabeth Martin

Adolph S. Flemister

See next page for additional authors

Follow this and additional works at: https://digitalcommons.georgefox.edu/pt_fac

Part of the Physical Therapy Commons 
Authors

Heather Barske, Ruth Chimenti, Josh Tome, Elizabeth Martin, Adolph S. Flemister, and Jeff Houck 


\title{
Clinical Outcomes and Static and Dynamic Assessment of Foot Posture After Lateral Column Lengthening Procedure
}

\author{
Heather Barske, MD, FRCSC', Ruth Chimenti, PT, DPT ${ }^{2}$, Josh Tome ${ }^{3}$, \\ Elizabeth Martin, MD ${ }^{4}$, Adolph S. Flemister, $\mathrm{MD}^{4}$, and Jeff Houck, PT, PhD $^{5}$
}

\begin{abstract}
Background: Lateral column lengthening (LCL) has been shown to radiographically restore the medial longitudinal arch. However, the impact of LCL on foot function during gait has not been reported using validated clinical outcomes and gait analysis.

Methods: Thirteen patients with a stage II flatfoot who had undergone unilateral LCL surgery and I3 matched control subjects completed self-reported pain and functional scales as well as a clinical examination. A custom force transducer was used to establish the maximum passive range of motion of first metatarsal dorsiflexion at $40 \mathrm{~N}$ of force. Foot kinematic data were collected during gait using 3-dimensional motion analysis techniques.

Results: Radiographic correction of the flatfoot was achieved in all cases. Despite this, most patients continued to report pain and dysfunction postoperatively. Participants post LCL demonstrated similar passive and active movement of the medial column when we compared the operated and the nonoperated sides. However, participants post LCL demonstrated significantly greater first metatarsal passive range of motion and first metatarsal dorsiflexion during gait than did controls $(P<.0$ I for all pairwise comparisons).

Conclusion: Patients undergoing LCL for correction of stage II adult-acquired flatfoot deformity experience mixed outcomes and similar foot kinematics as the uninvolved limb despite radiographic correction of deformity. These patients maintain a low arch posture similar to their uninvolved limb. The consequence is that first metatarsal movement operates at the end range of dorsiflexion and patients do not obtain full hindfoot inversion at push-off. Longitudinal data are necessary to make a more valid comparison of the effects of surgical correction measured using radiographs and dynamic foot posture during gait.

Level of Evidence: Level III, comparative series.
\end{abstract}

Keywords: flatfoot, lateral column lengthening, kinematics, gait analysis

Adult-acquired flatfoot deformity (AAFD) is a common clinical problem presenting to the orthopedic surgeon. The causes of adult-acquired flatfoot are multiple. The planovalgus deformity occurs secondary to dysfunction of the posteromedial soft tissues of the foot. Commonly, the posterior tibialis tendon becomes incompetent, leading to attenuation of the ligamentous support of the medial longitudinal arch. This results in peritalar subluxation with varying degrees of increased hindfoot valgus, flattening of the medial longitudinal arch, forefoot abduction, and forefoot varus. ${ }^{17,22,24}$

The repetitive microtrauma on the posteromedial hindfoot with activity causes pain and leads patients to seek medical attention. Treatment of AAFD is initially nonoperative. $^{17,22,24}$ If this fails, operative treatment is based on staging of the disease. Johnson and Strom ${ }^{18}$ originally categorized AAFD into 3 stages based on the extent of the deformity and the flexibility of the subtalar joint. Stage II disease is characterized by a flexible pes planovalgus deformity where the subtalar joint is flexible and the hindfoot valgus is correctable actively or passively. Controversy surrounds the surgical treatment of stage II disease. There is agreement that stage II flatfoot should be corrected with

\footnotetext{
'University of Manitoba, Winnipeg, MB, Canada

${ }^{2}$ University of Rochester, School of Nursing, Ithaca College, Department of Physical Therapy, Rochester, NY, USA

${ }^{3}$ Ithaca College Movement Analysis Laboratory, Rochester, NY, USA

${ }^{4}$ University of Rochester Medical Center, Department of Orthopaedics, Rochester, NY, USA

${ }^{5}$ Physical Therapy, Ithaca College, Rochester, NY, USA
}

\section{Corresponding Author:}

Heather Barske, MD, FRCSC, University of Manitoba, Surgery, 75

Poseidon Bay, Winnipeg, MB, R3M 3E4 Canada

Email: heatherbarske@yahoo.ca 
joint-sparing procedures; however, the best combination of soft tissue procedures and realignment osteotomies is not defined by the current literature. ${ }^{11}$

A lateral column lengthening (LCL) procedure combined with soft tissue techniques spares the joints of the foot while improving alignment. Evans ${ }^{6}$ first described the lateral column lengthening procedure in 1975. Sangeorzan et $\mathrm{al}^{26}$ applied the procedure to the stage II AAFD. Currently, an LCL is often chosen to correct the stage II flatfoot because it simultaneously corrects the hindfoot valgus and forefoot abduction, raises the medial longitudinal arch, and unloads the first metatarsocuneiform joint. ${ }^{5,11,26}$ It does this by adducting and plantar flexing the midfoot around the talar head. An LCL has been shown to provide greater realignment than a medializing calcaneal osteotomy. ${ }^{2}$ It is a popular choice for correction of the flexible flatfoot and has resulted in good patient outcomes. ${ }^{7,11,12}$ A survey of foot and ankle surgeons in 2002 revealed that $41 \%$ of surgeons performed lateral column lengthening as part of the bony procedure in the correction of a flatfoot. ${ }^{11}$

Despite more than 2 decades of use of the lateral column lengthening in AAFD, few data are available to inform patients about their expected status if they elect these procedures. A few studies have reported improvement in American Orthopaedic Foot \& Ankle Society (AOFAS) scores, but no studies have been performed using validated musculoskeletal outcome measures. ${ }^{12,21,28}$ General health scores such as the SF-12 and SF-36 have been used to assess patients after LCL. ${ }^{25,28}$ Alterations in alignment of the medial longitudinal arch on weight-bearing radiographs also have been assessed after LCL procedures. The lateral talus-first metatarsal angle or Meary's line has good interand intraobserver correlation and is used to assess collapse through the talonavicular, naviculocuneiform, and first tarsometatarsal joints. ${ }^{32}$ Normal values are within 7 degrees of neutral. ${ }^{32}$ Studies have reported correction of these radiographic angles following LCL., ${ }^{2,10}$

Untested in previous studies is the influence of LCL procedures on medial longitudinal arch motion, defined by passive range of motion (ROM) and walking foot kinematics of the first metatarsal. Although the LCL procedures are designed to spare joint function, there has been little emphasis on evaluating joint function after LCL procedures. Kinematic studies during gait show that persons with AAFD demonstrate greater hindfoot eversion, greater first metatarsal dorsiflexion (suggesting lowering of the medial longitudinal arch), and increased forefoot abduction compared with healthy controls. ${ }^{15,29}$

Weakness of the tibialis posterior muscle has been associated with these changes in foot kinematics. ${ }^{23}$ Recent work has also demonstrated greater first metatarsal dorsiflexion relative to the calcaneus during bilateral heel raises in people with AAFD. ${ }^{13,15}$ Although the functional consequences of these alterations in foot kinematics are unknown, improvement in radiographic alignment associated with LCL procedures may also influence passive ROM of the first metatarsal and hindfoot eversion kinematics during walking. Damage to ligaments that stabilize the midfoot in AAFD raises the possibility of greater passive ROM of the first metatarsal. Theoretically, the LCL procedure may tighten the medial foot ligaments, decreasing ROM and restricting foot kinematics during walking. To date there have been no studies addressing the influence of lateral column lengthening on passive ROM or forefoot kinematics during walking.

The purpose of this case-control study was to (1) clinically assess participants post LCL (in terms of maximum number of heel raises, foot posture, and radiographic angles); (2) assess self-reported function between participants post LCL and controls (using the visual analogue scale [VAS] for pain and Short Musculoskeletal Functional Assessment [SMFA]); and (3) compare foot ROM measures collected passively and during walking in participants post LCL and in controls. For the clinical assessment we hypothesized that heel raise ability and arch height index of the participants post LCL would be similar to those of controls. We also compared radiographic angles collected before and after surgery. We hypothesized that weight-bearing radiographic findings would demonstrate decreased talusfirst metatarsal angles after surgery compared with before surgery. The SMFA was used to compare self-reported function between participants post LCL and controls. We hypothesized that the SMFA scores and VAS pain ratings would approach data from controls. Last, we compared 3-dimensional motion analysis of the operated and nonoperated sides of the participants post LCL and controls. The focus was on passive and active (ie, walking) movement of the first metatarsal and active (ie, walking) movement of the calcaneus. We hypothesized that the participants post LCL would show similar movements when we compared the operated and nonoperated sides. However, because many patients with AAFD have a flatfoot posture bilaterally, we hypothesized that when the operated and nonoperated sides were compared with controls they would show greater passive first metatarsal ROM and actively greater first metatarsal dorsiflexion and greater hindfoot eversion, indicating a greater flatfoot.

\section{Material and Methods}

\section{Participants}

All patients who had undergone an LCL procedure between 2006 and 2009 for stage II AAFD by 1 of 2 fellowshiptrained foot and ankle surgeons were invited to participate. Subjects were excluded if they were unable to ambulate 50 feet without significant discomfort or difficulty, had a comorbid condition (eg, insensate feet, metatarsus primus varus) in the same foot as AAFD surgery, had a 
history of ipsilateral lower extremity pain or surgery not due to AAFD, or had a postoperative infection or other serious operative complication. Thirty-four patients were eligible for the study. Thirteen agreed to participate and completed the testing session (Table 1). All patients underwent an LCL combined with a flexor digitorum longus transfer to the navicular. For the LCL, iliac crest autograft was used in 11 of 13 patients; allograft iliac crest was used in 2 patients. Six patients had a triple hemisection Achilles tendon lengthening, and 6 had a gastrocnemius recession. Four patients had a first tarsometatarsal fusion at the time of their flatfoot reconstruction.

Control subjects were healthy individuals without foot or ankle abnormality or discomfort who were recruited from the general population. They were of similar age and gender as subjects with AAFD. The exclusion criteria described above were used. Control subjects completed the same self-report questionnaires and laboratory testing session with kinematic analysis as did subjects with AAFD. However, controls were not included in the radiographic analysis. Thirteen healthy volunteers participated. Control participants were statistically similar for age $(t=0.17, P=$ $.87)$ and gender $\left(\chi^{2}=1.18, P=.28\right)$ but not for body mass index $(t=2.52, P=.02)$ (Table 1). All subjects signed a consent form and were informed of the study procedures and risks consistent with an approved protocol by associated institutional review boards.

\section{Clinical Assessment}

All patients underwent a single laboratory testing session by an independent examiner that included a clinical assessment (maximum number of heel raises and arch height index), self-report measures (VAS pain and SMFA), and a movement analysis test. The number of heel raises each participant was able to perform was determined for each side. During testing each participant was required to achieve a height similar to that achieved in the initial attempt. The number of heel raises was stopped when the participants stopped, started bending their knee, or lost heel height compared with their initial attempt. The arch height index was used to determine the degree of pes planus deformity on the involved side as described by Williams and McClay. ${ }^{31}$ The arch height index is a ratio of the height of the dorsum of the foot divided by the length. The dorsum height is taken at $50 \%$ of the foot length, divided by the foot length from the heel to the base of the distal first metatarsal head. ${ }^{31}$ Greater values indicate a higher arch. Arch height index values can be compared with uninjured samples reported in the literature.

\section{Self-Reported Function}

To assess function, patients completed the SMFA questionnaire and a VAS for pain. To assess pain level, we asked each participant to rank his or her pain at its best, and worst, in the last week on a 0 to 10 scale, where 10 was the worst pain the participant could imagine. The SMFA is a 46-item self-report questionnaire. The questionnaire assesses the indices Function (25 items, including mobility [9 items]) and Bothersome (12 items). ${ }^{20,27}$ The scale was originally developed with 420 patients who had acute fracture or soft tissue injury. Content, convergent, and construct validity was demonstrated with clinical data, Short Form-36 results, and life-change data with very few ceiling effects and no floor effects reported. ${ }^{20,27}$ The extensive normative data that exist for the SMFA make the scale helpful to compare data from the proposed study with population norms. ${ }^{16}$ The SMFA is also particularly suitable for the current investigation given the presence of a subcategory of questions that pertains specifically to mobility.

\section{Radiographic Assessment}

Standing anteroposterior and lateral radiographic views of the affected foot were reviewed preoperatively and at latest follow-up. The long axis of the talus and first metatarsal was used to measure the lateral and anteroposterior talusfirst metatarsal angles. The weight-bearing lateral views were also used to assess naviculocuneiform sag, which was graded as present or absent. Although the presence or absence of "sag" has not been evaluated for reliability, it is included here because it is used clinically as an indicator of arch correction.

\section{First Metatarsal Range of Motion}

Passive ROM of the first metatarsal was assessed using a custom-designed force gauge motivated by previous devices. ${ }^{8,9}$ A force gauge (model SML-25 Interface, Scottsdale, Arizona) was calibrated using known weights $\left(r^{2}=0.977\right)$ and integrated into a custom jig to move the distal end of the first metatarsal (Figure 1). Pads connected in series with the force gauge were designed to fit around the first metatarsal head without disturbing the markers used to track movement during the walking analysis (Figure 1). To evaluate first metatarsal dorsiflexion and first metatarsal plantar flexion ROM, the following procedures were used. The participants were positioned seated with the leg vertical and foot resting on a step. The foot was placed with the second metatarsal on the edge of the step, allowing the force gauge to be placed on the first metatarsal head. The bottom pad was adjustable to accommodate the shape of the metatarsal head for each participant. Once the force gauge was adjusted for each participant, two 40-N upward (dorsiflexion) and downward (plantar flexion) loads were applied to each patient. An oscilloscope was used to display the force readings in real time. Using the oscilloscope feedback, the examiner 
Table I. Demographics

\begin{tabular}{|c|c|c|c|c|c|c|}
\hline LCL Subjects & Age, $y$ & Sex & $\mathrm{BMI}, \mathrm{kg} / \mathrm{m}^{2}$ & $\begin{array}{c}\text { Side } \\
\text { Involved }\end{array}$ & Procedures & $\begin{array}{l}\text { Time Since } \\
\text { Surgery, mo }\end{array}$ \\
\hline I & 66 & $\mathrm{~F}$ & 25.9 & Right & $\begin{array}{l}\text { FDL, LCL, ICBG, } \\
\text { GR, first TMT }\end{array}$ & 18 \\
\hline 2 & 65 & $F$ & 29.3 & Left & $\begin{array}{l}\text { FDL, LCL, ICBG, } \\
\text { GR, first TMT }\end{array}$ & 35 \\
\hline 3 & 50 & $\mathrm{~F}$ & 26.6 & Left & $\begin{array}{l}\text { FDL, LCL, ICBG, } \\
\text { GR, first TMT }\end{array}$ & 21 \\
\hline 4 & 72 & $\mathrm{~F}$ & 27.1 & Left & $\begin{array}{l}\text { FDL, LCL, ICBG, } \\
\text { TAL, first TMT }\end{array}$ & 56 \\
\hline 5 & 60 & $\mathrm{~F}$ & 27.8 & Left & $\begin{array}{l}\text { FDL, LCL, ICBG, } \\
\text { TAL, Spring }\end{array}$ & 11 \\
\hline 6 & 60 & $\mathrm{~F}$ & 38.3 & Right & $\begin{array}{c}\text { FDL, LCL, ICBG, } \\
\text { TAL }\end{array}$ & 28 \\
\hline 7 & 62 & $\mathrm{~F}$ & 29.3 & Left & $\begin{array}{l}\text { FDL, LCL, GR, } \\
\text { Spring,Allog }\end{array}$ & 19 \\
\hline 8 & 51 & M & 32.3 & Left & $\begin{array}{c}\text { FDL, LCL, ICBG, } \\
\text { GR }\end{array}$ & 14 \\
\hline 9 & 59 & $\mathrm{~F}$ & 44.9 & Right & $\begin{array}{l}\text { FDL, LCL, Allog, } \\
\text { MCL }\end{array}$ & 27 \\
\hline 10 & 71 & $\mathrm{~F}$ & 30.0 & Left & $\begin{array}{c}\text { FDL, LCL, ICBG, } \\
\text { GR }\end{array}$ & 48 \\
\hline II & 52 & $F$ & 32.5 & Right & $\begin{array}{c}\text { FDL, LCL, ICBG, } \\
\text { TAL }\end{array}$ & 12 \\
\hline 12 & 48 & $F$ & 21.3 & Left & $\begin{array}{c}\text { FDL, LCL, ICBG, } \\
\text { TAL }\end{array}$ & 21 \\
\hline 13 & 35 & $F$ & 54.4 & Right & $\begin{array}{c}\text { FDL, LCL, ICBG, } \\
\text { TAL }\end{array}$ & 10 \\
\hline $\operatorname{LCL}_{\text {group }}{ }^{\mathrm{a}}(\mathrm{n}=13)$ & $57.8 \pm 10.3$ & $92 \% \mathrm{~F}$ & $32.3 \pm 8.9$ & $62 \%$ Left & NA & $24.6 \pm 14.2$ \\
\hline Controls $^{\mathrm{a}}(\mathrm{n}=13)$ & $57.2 \pm 5.4$ & $77 \% \mathrm{~F}$ & $25.5 \pm 3.8$ & NA & NA & NA \\
\hline Statistical value, $P$ value & $t=0.17, P=.87^{b}$ & $\chi^{2}=1.18, P=.28^{c}$ & $t=2.52, P=.02^{\mathrm{b}}$ & NA & NA & NA \\
\hline
\end{tabular}

Abbreviations: Allog, iliac crest allograft; BMI, body mass index; F, female; FDL, flexor digitorum longus transfer to the navicular; first TMT, first tarsometatarsal fusion; GR, gastrocnemius recession; ICBG, iliac crest bone graft; LCL, lateral column lengthening; $M$, male; $M C L$, medial collateral ligament repair; NA, not applicable; Spring, spring ligament repair;TAL, triple hemisection tendo-Achilles lengthening.

${ }^{a}$ Data presented as mean \pm standard deviation unless otherwise indicated.

bIndependent samples $t$ test.

${ }^{\mathrm{C}} \mathrm{C}$ i-square test for independence.

was able to hold the $40-\mathrm{N}$ positions (dorsiflexion or plantar flexion) for 1 to 2 seconds. During this 1 - to 2 -second interval, 1 second of kinematic data were collected (see description below). The sequence of applying the dorsiflexion and plantar flexion 40-N load was random, varying for each participant, in order to minimize sequence effects. Prior to the start of data collection, 4 control participants repeated this procedure on separate days to determine reliability. The between-day correlation coefficients were 0.99 and 0.83 for dorsiflexion and plantar flexion, respectively. The range of errors between days was 0.1 to 1.0 degrees for dorsiflexion and 1.1 to 3.1 degrees for plantar flexion.

\section{Foot Kinematic Measurements}

Foot kinematics were collected to determine first metatarsal movement in the sagittal plane (dorsiflexion) and hindfoot movement in the frontal plane (eversion). The foot segments measured were the tibia, calcaneus, first metatarsal, second to fourth metatarsals, and hallux. Infrared emitting diodes were mounted on thermoplastic molded platforms and placed directly on the skin overlying the calcaneus (hindfoot segment), first metatarsal (first metatarsal segment), second to fourth metatarsals, and hallux (Figure 2). The hallux and second to fourth metatarsal segment data were not used in this analysis. Placement of the thermoplastic platforms was 


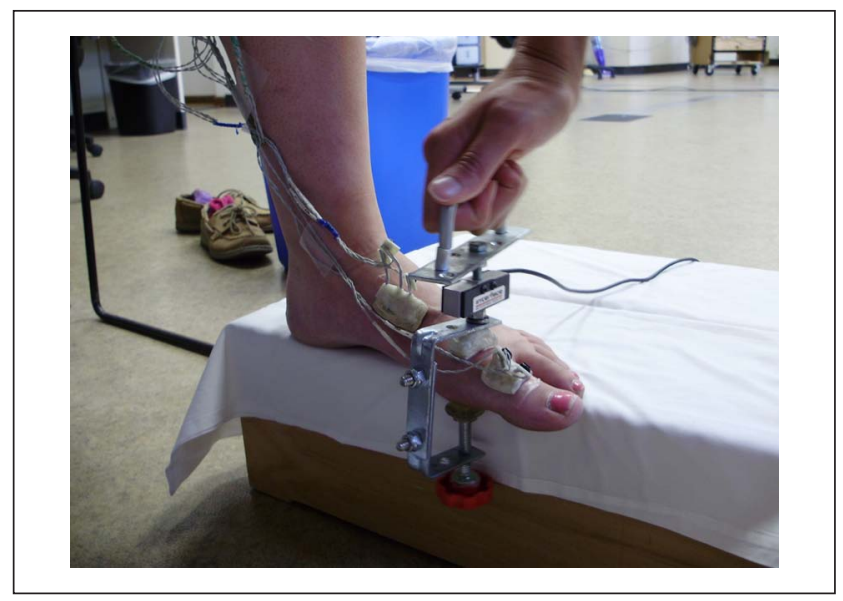

Figure I. Force gauge mounted on the first metatarsal head to apply a $40-\mathrm{N}$ superior(dorsiflexion) and downward (plantar flexion) force.

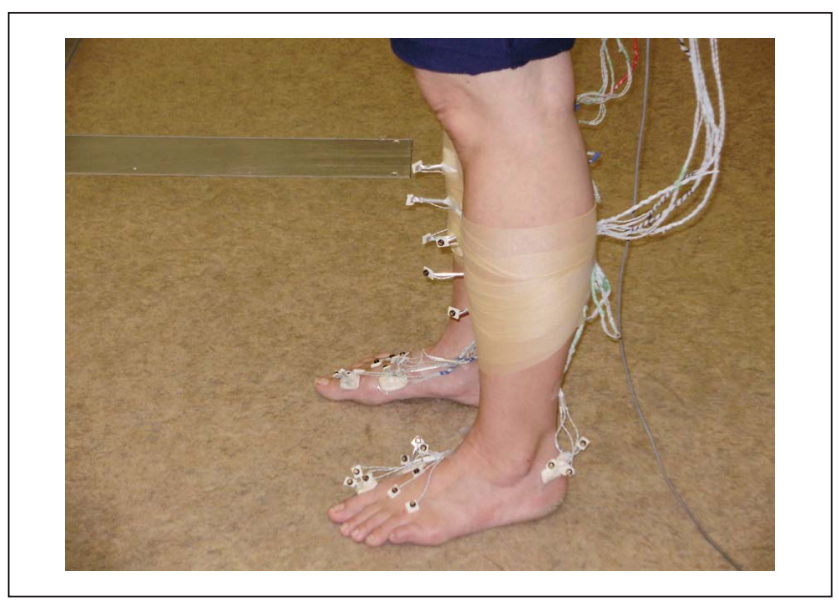

Figure 2. Infrared emitting diodes on thermoplastic molded platforms placed overlying the calcaneus, first metatarsal, second through fourth metatarsals, and hallux.

based on previous studies that showed good repeatability and validity of tracking the hindfoot and first metatarsal segments using skin-mounted sensors. ${ }^{14,30}$ Foot segment movement was tracked at $60 \mathrm{~Hz}$ using a 6-camera Optrotrak Motion Analysis System (Northern Digital, Waterloo, Canada). Data processing included smoothing the kinematic signal using a fourth-order, zero phase lag, Butterworth filter with a cut-off frequency of $6 \mathrm{~Hz}$.

Segment angles were determined by first referencing rigid body representation of each segment to digitized bony landmarks consistent with previous studies. ${ }^{15,23,29}$ The conventions used result in a right-hand Cartesian reference system for each segment (tibia, hindfoot, and first metatarsal). Once reference frames were established, a Z-X-Y sequence of rotations was used to calculate 2 angles: first metatarsal dorsiflexion/plantar flexion with respect to the hindfoot, and hindfoot inversion/eversion with respect to the tibia. The first metatarsal plantar flexion/dorsiflexion angle was a rotation around a medial-lateral axis (ie, motion in sagittal plane). The hindfoot inversion/eversion angle was a rotation around an anterior-posterior axis (ie, motion in frontal plane).

Participants were asked to walk down a $10-\mathrm{m}$ walkway at a speed of $1 \mathrm{~m} / \mathrm{s}$ to capture foot kinematics. To ensure that participants walked at the target speed, speed was monitored with the use of a timing system (Brower, Salt Lake City, Utah) and maintained during testing to within $\pm 5 \%$ of the target speed of $1 \mathrm{~m} / \mathrm{s}$. This slow walking speed was used to accommodate subjects with more severe problems. At a 10-N threshold, an embedded force plate (model 9286, Kistler, Switzerland) was used to identify initial contact and toe-off points during stance with force data collected at $1000 \mathrm{~Hz}$. Each subject completed a minimum of 5 successful trials consisting of full contact with the force plate.

\section{Analysis}

To assess the proposed hypotheses, a variety of statistical analyses were used. For the clinical assessment variables, 2 -sided independent samples $t$ tests were used to compare the means of the participants post LCL to controls for the best/worst pain score. One-way analyses of variance (ANOVAs) were used to assess differences between the 3 groups (LCL involved side, LCL uninvolved side, controls) in the maximum number of single-limb heel raises and the arch height index. If there were significant differences between the 3 groups with the 1-way ANOVA, then pairwise comparisons were used to test for significant differences among LCL involved side, LCL uninvolved side, and controls. Repeated-measures $t$ tests were used to compare radiographic angles before and after surgery.

A mixed-effects 2-way ANOVA was used to examine for differences in self-reported function between groups (LCL and controls) by SMFA subscore. The fixed factor was group with 2 levels (LCL and controls). The random factor was SMFA subscore with 3 levels (Function, Mobility, and Bothersome). The presence of interaction effects was determined prior to assessing main effects. A main effect, indicating higher scores in the LCL group (ie, worse function) across all SMFA subscores, would result in a rejection of the initial hypotheses that the LCL group would report similar function as controls.

Prior to analysis of the first metatarsal data, preliminary analyses were completed. Because some patients received a first tarsometatarsal fusion, this subgroup was initially analyzed separately to assess the effect on first metatarsal ROM. Descriptive data (Tables 1 and 2) and an independent-samples $t$ test demonstrated that there were no significant differences in passive first metatarsal ROM between 
Table 2. Radiographic Measures

\begin{tabular}{|c|c|c|}
\hline \multirow[b]{2}{*}{ LCL Subjects } & \multicolumn{2}{|c|}{$\begin{array}{l}\text { Decrease in Talus-First } \\
\text { Metatarsal Angle, deg }\end{array}$} \\
\hline & APView & Lateral View \\
\hline I & 27 & 16 \\
\hline 2 & 10 & 38 \\
\hline 3 & - & - \\
\hline 4 & 8 & 21 \\
\hline 5 & 25 & 33 \\
\hline 6 & 25 & 28 \\
\hline 7 & 29 & 7 \\
\hline 8 & 14 & 17 \\
\hline 9 & 18 & 18 \\
\hline 10 & 5 & II \\
\hline II & - & - \\
\hline 12 & 13 & 8 \\
\hline 13 & 11 & 17 \\
\hline $\begin{array}{l}\text { LCL group involved } \\
\operatorname{side}^{\mathrm{a}}(\mathrm{n}=13)\end{array}$ & $17.5 \pm 16.6$ & $15.4 \pm 10.4$ \\
\hline $\begin{array}{l}\text { LCL group uninvolved } \\
\operatorname{side}^{\mathrm{a}}(\mathrm{n}=12)\end{array}$ & NA & NA \\
\hline Controls $^{a}(n=13)$ & NA & NA \\
\hline Statistical value, $P$ value & $t=6.64, P<.0 \mathrm{I}^{\mathrm{b}}$ & $t=6.64, P<.0 \mathrm{I}^{\mathrm{b}}$ \\
\hline
\end{tabular}

Abbreviations: AP, anteroposterior; $L C L$, lateral column lengthening; NA, not applicable, - , data not collected.

a Data presented as mean \pm standard deviation.

${ }^{\mathrm{b}}$ Repeated measures $t$ test.

people with $(\mathrm{n}=4)$ and without $(\mathrm{n}=9)$ a first tarsometatarsal fusion $(t=0.06, P=.96)$. Because of the small sample, this analysis does not rule out a subtle effect of the first tarsometatarsal fusion on ROM, yet large effects were not evident. Thus, for subsequent analyses, the LCL involved group included both people with and without a first tarsometatarsal fusion. Because there were no significant differences between sides for the control group for all passive ROM and walking kinematics (paired t tests, $P>.05$ for all comparisons), the left side was arbitrarily chosen as the involved side for the control group.

One-way ANOVAs were used to compare the average passive ROM variables between the 3 groups (LCL involved, LCL uninvolved, and controls). The average of the maximum first metatarsal plantar flexion/dorsiflexion from the two 40-N trials was used in the analysis. In addition, the difference between the maximum first metatarsal dorsiflexion and first metatarsal plantar flexion was computed to evaluate the total first metatarsal passive ROM. A 1-way ANOVA was used to compare first metatarsal plantar flexion passive ROM between the LCL involved, LCL uninvolved, and controls. The same 1-way ANOVA procedures were used for first metatarsal dorsiflexion. For each dependent variable, in the presence of a significant main effect of a 1-way ANOVA, pairwise comparisons were performed to detect differences among the 3 groups (LCL involved, LCL uninvolved, and controls). A significant main effect followed by significant pairwise comparisons indicating greater first metatarsal dorsiflexion of the LCL involved and LCL uninvolved groups compared with controls was consistent with a hypothesis of increased forefoot mobility.

One-way ANOVA models were also used to evaluate foot kinematic data during walking. Initially, walking trials were time normalized to $100 \%$ of stance, resulting in 101 points for each walking trial across stance. Subsequently, a minimum of 3 trials were averaged for each kinematic variable to gain a representative pattern for each subject. Key points of gait were chosen to reflect patterns of first metatarsal plantar flexion/dorsiflexion (initial contact, $12 \%$ of stance, $78 \%$ of stance, toe-off) and hindfoot inversion/eversion (initial contact, $25 \%$ of stance, $92 \%$ of stance, toe-off). ${ }^{15}$ For each key point of gait, for each dependent variable a 1-way ANOVA was used to test for group main effect. If significant, this was followed by pairwise comparisons to determine which groups differed (LCL involved, LCL uninvolved, and controls). As with the passive ROM variables, we hypothesized that there would be a main effect of group for all gait ROM variables. Furthermore, we hypothesized that pairwise comparisons would demonstrate no differences between the involved and uninvolved side of the LCL groups and that both LCL groups would have a greater amount of passive ROM than the control group. All data analyses were performed using SPSS 17.0 for Windows (SPSS Inc, Chicago, Illinois). Statistical significance was defined as a 2-tailed $P$ value $\leq .05$ for all analyses.

\section{Results}

\section{Clinical Assessment}

The number of single heel raises was significantly different among groups; however, the arch height index was not different among groups. Controls demonstrated a significantly higher number of single-limb heel raises than the LCL involved $(P<.01)$ and LCL uninvolved sides $(P<.01)$. However, there was no difference from the LCL involved to the LCL uninvolved side $(P=.2)$. A 1-way ANOVA indicated that there were no significant differences in arch height index between the LCL involved, LCL uninvolved, and control groups $(P=.11)$ (Table 3$)$.

\section{Self-Reported Function}

There were significant differences in the VAS pain and SMFA scores. The best VAS pain scores were not different between the LCL and control groups $(P=.15)$. However, the LCL group reported greater pain on the VAS for worst 
Table 3. Clinical Measures, Self-Reported Pain, and Function

\begin{tabular}{|c|c|c|c|c|c|c|c|}
\hline \multirow[b]{2}{*}{ LCL Subjects } & \multirow[b]{2}{*}{ Arch Height Index } & \multirow{2}{*}{$\begin{array}{l}\text { Max No. Single- } \\
\text { Limb Heel Raises }\end{array}$} & \multirow[b]{2}{*}{ VAS Best } & \multirow[b]{2}{*}{ VASWorst } & \multicolumn{3}{|c|}{ SMFA } \\
\hline & & & & & Function & Mobility & Bothersome \\
\hline 1 & 0.15 & 12 & 0 & 0 & 5.9 & 0 & 6.3 \\
\hline 2 & 0.27 & 0 & 0 & 8 & 16.2 & 25 & 8.3 \\
\hline 3 & 0.35 & 0 & 0 & 6 & 25.0 & 30.6 & 37.5 \\
\hline 4 & 0.31 & 0 & 0 & 0 & 18.4 & 16.7 & 10.4 \\
\hline 5 & 0.27 & 0 & 0 & 4 & 4.4 & 2.8 & 10.4 \\
\hline 6 & 0.24 & 20 & 0 & 0 & 16.2 & 25 & 12.5 \\
\hline 7 & 0.32 & 5 & 0 & I & 16.9 & 22.2 & 18.8 \\
\hline 8 & 0.31 & 6 & 0 & I & 22.8 & 30.6 & 25.0 \\
\hline 9 & 0.3 & 0 & 3 & 8 & 25.0 & 41.7 & 14.6 \\
\hline 10 & 0.27 & 0 & 0 & - & 13.2 & 16.7 & 22.9 \\
\hline 11 & 0.33 & 3 & 2 & 5 & 30.2 & 33.3 & 43.8 \\
\hline 12 & 0.36 & 7 & 0 & I & 2.9 & 8.3 & 0 \\
\hline 13 & 0.30 & 0 & 8 & 10 & 44.1 & 72.2 & 41.7 \\
\hline $\begin{array}{l}\text { LCL group involved } \\
\operatorname{side}^{a}(n=13)\end{array}$ & $0.29 \pm 0.05$ & $4.1 \pm 6.1$ & $1.1 \pm 2.4$ & $3.7 \pm 3.7$ & $18.6 \pm 11.3$ & $25.0 \pm 18.7$ & $19.4 \pm 14.0$ \\
\hline $\begin{array}{l}\text { LCL group uninvolved } \\
\operatorname{side}^{a}(n=12)\end{array}$ & $0.29 \pm 0.05$ & $8.3 \pm 6.6$ & NA & NA & NA & NA & NA \\
\hline Controls $^{a}(n=13)$ & $0.33 \pm 0.03$ & $25.8 \pm 5.7$ & $0.0 \pm 0.0$ & $0.7 \pm 1.5$ & $1.83 \pm 1.42$ & $1.9 \pm 2.5$ & $0.9 \pm 1.4$ \\
\hline Statistical value, $P$ value & $F=2.34, P=.1 I^{b}$ & $F=83.73, P<.0 I^{b}$ & $t=1.57, P=.15^{c}$ & $\begin{array}{l}t=2.63 \\
P=.02^{c}\end{array}$ & \multicolumn{3}{|c|}{$\begin{array}{l}\text { Main effect for groups }{ }^{\mathrm{d}}: F=23.69 \\
\qquad P<.01\end{array}$} \\
\hline
\end{tabular}

Abbreviations: AP, anteroposterior; LCL, lateral column lengthening; NA, not applicable; SFMA, Short Form Musculoskeletal Assessment; VAS, visual analogue scale; - , data not collected.

a'Data presented as mean \pm standard deviation.

${ }^{b}$ One-way analysis of variance. Pairwise comparisons: $L C L$ involved to uninvolved, $P=.20$; $L C L$ involved to controls, $P<.01$; $L C L$ uninvolved to controls, $P<.01$.

'Independent samples $t$ test.

${ }^{\mathrm{d}}$ Result of 2-way mixed effect analysis of variance comparing across LCL and controls.

pain than did controls $(P=.02)$. The LCL group reported significantly greater scores on the SMFA compared with healthy adults across the subscales. There was no interaction between group and SMFA subscale $(P=.12)$. However, higher scores (Table 3 ) across subscales resulted in a main effect for group $(P<.01)$. Pairwise comparisons indicated that all 3 scales were significantly higher compared with controls $(P<.01$ for Function, Mobility, and Bothersome) (Table 3).

\section{Radiographic Assessment}

Radiographic measures were significantly improved after LCL $(P<.01$ for both angles compared pre- to postsurgery) (Table 2). Average correction of the lateral talus-first metatarsal angle was $15.4 \pm 10.4$ degrees and average correction of the AP talus-first metatarsal angle was $17.5 \pm$ 16.6 degrees (Table 2). The preoperative lateral talusfirst metatarsal angle improved from $25.6 \pm 15.7$ degrees to $5.8 \pm 7.5$ degrees. Six patients with naviculocuneiform sag demonstrated improvement of the sag on postoperative weight-bearing films.

\section{First Metatarsal Range of Motion}

There were significant differences between groups in maximum first metatarsal passive dorsiflexion and total first metatarsal passive ROM (Table 4). There were significant differences across groups for first metatarsal dorsiflexion $\operatorname{ROM}(P<.01)$ and maximum first metatarsal dorsiflexion $(P<.01)$. Both the involved and uninvolved sides of the LCL group demonstrated significantly greater maximum first metatarsal dorsiflexion ROM and total first metatarsal passive ROM compared with controls (pairwise comparisons for first metatarsal dorsiflexion ROM: LCL involved vs control, $P<.01$, LCL uninvolved vs control, $P=$ .01 ; for first metatarsal total passive ROM: LCL involved vs control, $P<.01$, LCL uninvolved vs control, $P<.01$ ). There were no significant differences between the involved and uninvolved sides for the LCL group $(P \geq .30)$. There 
Table 4. Passive Range of Motion With $40 \mathrm{~N}$ of Pressure

\begin{tabular}{|c|c|c|c|}
\hline LCL Subjects & $\begin{array}{c}\text { Maximum Passive DF, deg } \\
\text { (-plantar flexion/+dorsiflexion) }\end{array}$ & $\begin{array}{c}\text { Maximum Passive PF, deg } \\
\text { (-plantar flexion/+dorsiflexion) }\end{array}$ & Total Passive ROM, deg \\
\hline I & -15.3 & -26.0 & 10.7 \\
\hline 2 & -7.7 & -31.0 & 23.3 \\
\hline 3 & -17.3 & -32.1 & 14.8 \\
\hline 4 & -25.6 & -42.5 & 16.9 \\
\hline 5 & -12.9 & -30.6 & 17.8 \\
\hline 6 & -23.9 & -36.0 & 12.1 \\
\hline 7 & -11.2 & -34.1 & 22.9 \\
\hline 8 & -7.6 & -15.1 & 7.5 \\
\hline 9 & -5.3 & -18.5 & 13.2 \\
\hline 10 & -5.6 & -15.3 & 9.7 \\
\hline II & -3.0 & -32.1 & 29.1 \\
\hline 12 & -10.8 & -34.0 & 23.2 \\
\hline 13 & -15.5 & -29.8 & 14.2 \\
\hline LCL group involved side ${ }^{a}(n=13)$ & $-12.4 \pm 7.0$ & $-29.0 \pm 8.2$ & $16.6 \pm 6.4$ \\
\hline $\mathrm{LCL}_{\text {group uninvolved side }} \mathrm{a}(\mathrm{n}=12)$ & $-15.1 \pm 7.5$ & $-31.6 \pm 7.5$ & $16.5 \pm 5.5$ \\
\hline Controls $^{a}(n=13)$ & $-22.5 \pm 6.0$ & $-32.3 \pm 7.9$ & $9.8 \pm 3.9$ \\
\hline One-way ANOVA results & $F=7.64, P<.01$ & $F=0.63, P=.54$ & $F=6.80, P<.01$ \\
\hline
\end{tabular}

Abbreviations: ANOVA, analysis of variance; DF, dorsiflexion; LCL, lateral column lengthening; PF, plantar flexion; ROM, range of motion.

${ }^{a}$ Data presented as mean \pm standard deviation.

were no significant differences between groups in maximum first metatarsal plantar flexion $(P=.54)$.

\section{Foot Kinematic Measurements}

There were significant differences between groups in foot kinematics during walking (Table 4). For the key points of stance, first metatarsal dorsiflexion/plantar flexion was significantly different between groups. The LCL group had significantly greater first metatarsal dorsiflexion than controls for all 4 selected points of walking (pairwise comparisons: $P<.01$ for all 4 stance points). However, in comparisons across the involved and uninvolved side of the LCL group, there were no significant differences (pairwise comparisons: initial contact, $P=.73 ; 12 \%$ of stance, $P=$ $.70 ; 78 \%$ of stance, $P=.50$; toe-off, $P=.26$ ). In contrast to first metatarsal plantar flexion/dorsiflexion, the hindfoot eversion/inversion showed significance only at $92 \%$ of the stance phase of walking (Table 5). Controls demonstrated significantly more inversion than both LCL groups at $92 \%$ of stance (pairwise comparisons: LCL involved vs control, $P=.03$; LCL uninvolved vs control, $P=.03$ ).

\section{Discussion}

The purpose of this case-control study was to assess participants post LCL by clinical examination, self-reported functional scores, radiographs, and foot ROM measures collected passively and during gait. The findings of this study suggest that patients undergoing the combination of LCL and other soft tissue surgeries for correction of stage II AAFD experience mixed outcomes and similar foot kinematics as the uninvolved limb despite radiographic correction of deformity. Consistent with our clinical assessment hypotheses, the average VAS for best pain rating was similar between participants post LCL and controls. However, inconsistent with our clinical assessment hypotheses were increased VAS worst pain rating and greater difficulty in heel raise ability compared with controls. Participants post LCL demonstrated good foot alignment with a similar average arch height index as controls and decreased talus-first metatarsal angles after surgery compared with before surgery.

Further insight into the global status of patients following LCL surgery was obtained by the laboratory clinical examination (Table 1). Most patients fared poorly on the single heel raise test compared with control subjects. For healthy sedentary Taiwanese women aged 41 to 60 years and 61 to 80 years, a typical number of single-limb heel raises was previously reported to be $9.3 \pm 3.6$ and $2.7 \pm 1.5$, respectively. ${ }^{17}$ The sedentary sample and strict ROM criteria used in this study (ie, an electronic goniometer was used) may have led to lower values. Our control group vastly exceeded this estimation of typical heel raise ability, with the lowest number of heel raises being 14. If we classify participants post LCL according to the values by Jan et al, ${ }^{17}$ then 5 participants $(1,6,7,8$, and 12$)$ were able to achieve a typical number of heel raises (3); however, the 
Table 5. First Metatarsal and Calcaneal Motion During Gait

\begin{tabular}{|c|c|c|c|c|}
\hline & LCL Involved, deg & LCL Uninvolved, deg & Controls, deg & One-Way ANOVA Results \\
\hline \multicolumn{5}{|c|}{ First metatarsal motion ${ }^{\mathrm{a}}$ (-plantar flexion/+dorsiflexion) } \\
\hline Initial contact & $-|5.7| \pm 7.2 \mid$ & $-16.67 \pm 6.99$ & $-26.60 \pm 6.06$ & $F=10.23, P<.01$ \\
\hline $12 \%$ of stance & $-|3.4| \pm 7.38$ & $-|4.5| \pm 7.79$ & $-24.51 \pm 5.74$ & $F=9.82, P<.01$ \\
\hline $78 \%$ of stance & $-8.50 \pm 7.75$ & $-6.47 \pm 8.15$ & $-17.97 \pm 6.04$ & $F=8.89, P<.01$ \\
\hline Toe-off & $-23.53 \pm 8.53$ & $-28.00 \pm 12.09$ & $-34.84 \pm 8.64$ & $F=4.37, P=.02$ \\
\hline \multicolumn{5}{|c|}{ Calcaneal motion ${ }^{\mathrm{a}}$ (-eversion/+inversion) } \\
\hline Initial contact & $-3.61 \pm 2.78$ & $-4.03 \pm 4.59$ & $-1.78 \pm 2.36$ & $F=1.63, P=.21$ \\
\hline $25 \%$ of stance & $-5.73 \pm 3.26$ & $-7.20 \pm 3.65$ & $-4.59 \pm 2.44$ & $F=2.16, P=.13$ \\
\hline $92 \%$ of stance & $-0.32 \pm 3.08$ & $-0.44 \pm 4.75$ & $2.73 \pm 2.46$ & $F=3.325, P=.05$ \\
\hline Toe-off & $0.81 \pm 3.26$ & $0.97 \pm 4.80$ & $1.92 \pm 2.70$ & $F=0.34, P=.71$ \\
\hline
\end{tabular}

Abbreviations:ANOVA, analysis of variance; LCL, lateral column lengthening.

${ }^{\mathrm{a}}$ Data presented as mean \pm standard deviation.

participants' results are grossly lower than those of the controls used in this study (LCL group average $4.1 \pm 6.1$ compared with the control average $25.8 \pm 5.7$ ).

Few studies report functional outcomes after LCL. Hintermann et $\mathrm{al}^{12}$ reported improvement in the AOFAS score from 47 to 91 by 2 years postoperatively. ${ }^{12}$ MosierLaClaire et $\mathrm{al}^{21}$ and Tellisi et $\mathrm{al}^{28}$ reported similar improvement with the AOFAS. Tellisi et $\mathrm{al}^{28}$ reported a mean postoperative SF-36 score of 79.2 for patients who had undergone flatfoot reconstruction and correlated this to the AOFAS. Although we cannot compare our data with the data from these previous studies as different measures were used, we report reasonable outcomes following LCL. Using the VAS, 10 patients reported that, at best, their pain level was 0 out of 10 . Conversely, only 3 patients reported 0 out of 10 pain at worst. The self-reported function hypothesis that SMFA scores of participants post LCL would approach the scores of controls was partially supported. The SMFA subscales suggest mild functional difficulties and bothersomeness at an average follow-up of 24 months; however, individual responses varied. Patients reported average SMFA scores of 18.6, 25.0, and 19.4 for Function, Mobility, and Bothersome indexes respectively. These values represent patients indicating that they find mobility and function "a little difficult" and are "a little bothered" by most activities. Although these values are significantly higher than those of our control subjects indicating worse function, they are within the range of normative data that have been reported for the SMFA. ${ }^{16}$

A primary goal of the LCL surgery is to alter foot alignment. Radiographic data suggest that all LCL subjects had a decrease in the talus-first metatarsal angle on both the anterior-posterior and lateral radiographic views (Table 2). Similar to our results, previous authors have reported 12 to 20 degrees of correction with an LCL. ${ }^{2,4,12}$ All 6 patients who had naviculocuneiform sag on their preoperative lateral films demonstrated improvement or loss of the sag on postoperative weight-bearing films. It is also interesting to note that although no direct manipulation was performed on the naviculocuneiform joint during the surgical procedure, all patients who had naviculocuneiform sag on their preoperative films demonstrated improvement or full correction of the midfoot sag postoperatively. This is consistent with previous findings that an LCL adducts and plantar flexes the midfoot relative to the hindfoot, raises the medial longitudinal arch, and unloads the first metatarsocuneiform joint. ${ }^{5,26}$

Our hypothesis that the participants post LCL would show similar movements when we compared the operated and nonoperated sides was supported by both passive measures of first metatarsal ROM and hindfoot eversion/inversion. The hypothesis that the operated and nonoperated sides would demonstrate greater passive first metatarsal ROM and flatfoot posture than controls was supported. In the LCL patients, the total first metatarsal ROM was larger and shifted toward dorsiflexion during walking compared with controls (Table 4). This shows that patients with flatfeet operate at the end range of dorsiflexion of the first metatarsal relative to the calcaneus. This may represent hypermobility of the medial column that has occurred because of the flattened arch. Previous studies have shown that the functional ROM of patients with flatfeet occurs in a dorsiflexed range relative to controls. ${ }^{13,15}$ However, previous studies did not assess first metatarsal dorsiflexion/plantar flexion ROM. Unique to this study, measured passive ROM of the first metatarsal with a $40-\mathrm{N}$ force allowed the walking data to be referenced to the first metatarsal dorsiflexion/ plantar flexion ROM present. The foot walking kinematics demonstrated that patients who had undergone LCL surgery functioned toward the dorsiflexion limits of their available ROM, with some exceeding their maximum passive dorsiflexion by a few degrees. The same was true of the walking kinematics of their uninvolved side. These patients, however, did not reach their maximum plantar flexion during walking. In contrast, the controls met or exceeded both the 
maximum dorsiflexion and plantar flexion measurements during walking. The differences between the LCL groups and control subjects indicate that although the foot position is improved, patients are only restored to their contralateral equivalent, not to normal foot kinematics. The participants may have exceeded the passive ROM measurements due to increased forces applied across the midfoot during walking.

In contrast to first metatarsal dorsiflexion/plantar flexion, there were fewer significant differences between groups for calcaneal motion (hindfoot eversion/inversion) during gait (Table 5). Hindfoot eversion/inversion was not significantly different until late stance when the forefoot was loaded during push-off. The failure of the hindfoot to fully invert at $92 \%$ may influence first metatarsal kinematics. Hindfoot inversion is thought to contribute to arch raising and stability of the medial longitudinal $\mathrm{arch},{ }^{1}$ both of which may be associated with terminal plantar flexion of the first metatarsal. Brodsky et $\mathrm{al}^{3}$ also found no difference in hindfoot motion between flatfoot reconstruction (flexor digitorum longus transfer to the navicular, spring ligament imbrication, and medializing calcaneal osteotomy) and unaffected side or controls. ${ }^{3}$ Previous case-control studies of participants with posterior tibial tendon dysfunction prior to surgery suggest significantly larger hindfoot eversion. ${ }^{14,29}$ The hypothesis that nearly equivalent side-to-side hindfoot kinematics represent improvement is supported by one prospective study. Marks et $\mathrm{al}^{19}$ found that after an LCL, patients demonstrated increased hindfoot inversion during stance. Although more data on foot kinematics are desirable, the current studies provide preliminary evidence that walking kinematics are preserved post LCL surgery when combined with other procedures. The importance of restoring foot kinematics to the uninvolved side or controls on clinical outcomes remains theoretical.

The strengths of this study include the use of validated outcome measures and in-depth foot biomechanical analysis determined by an independent evaluator. This is the first study to report validated musculoskeletal outcome measures post LCL. There are several limitations to this study. The sample size is low; however, this is comparable to other studies that included motion analysis of patients with AAFD. ${ }^{3,13,15,19}$ Although the LCL group was similar in age and gender, the control group had a lower mean body mass index than the LCL group. The groups also varied considerably with regard to additional soft tissue and bony procedures that were combined with the LCL technique (Table 1). Remarkably, there was no obvious difference between patients who had undergone a first tarsometatarsal fusion and those who had not. This likely reflects the limitations of our foot modeling approach. Relative movement between the talonavicular, naviculocuneiform, and first tarsometatarsal joint is not measurable with the model we used in this study. Rather the first metatarsal dorsiflexion/plantar flexion kinematics used in this study are a composite of all these joints. The alterations in ROM attributable to the first tarsometatarsal fusion are likely occurring in these joints that we did not model. Another limitation is that preoperative motion analysis and functional scoring were not performed. It is difficult to interpret the SMFA and VAS scores without preoperative data.

\section{Conclusion}

Some patients may continue to have pain and disability with daily activities following surgical correction of their flatfoot with an LCL. These patients maintain a low arch posture similar to their contralateral foot and therefore operate at the end range of dorsiflexion for the first metatarsal and do not obtain full hindfoot inversion at push-off.

\section{Declaration of Conflicting Interests}

The author(s) declared no potential conflicts of interest with respect to the research, authorship, and/or publication of this article.

\section{Funding}

The author(s) received no financial support for the research, authorship, and/or publication of this article.

\section{References}

1. Blackwood CB, Yuen TJ, Sangeorzan BJ. The midtarsal joint locking mechanism. Foot Ankle Int. 2005;26(12):1074-1080.

2. Bolt P, Coy S, Toolan B. A comparison of lateral column lengthening and medial translational osteotomy of the calcaneus for the reconstruction of adult acquired flatfoot. Foot Ankle Int. 2007;28(11):1115-1123.

3. Brodsky J, Charlick D, Coleman S, Pollo F, Royer C. Hindfoot motion following reconstruction for posterior tibial tendon dysfunction. Foot Ankle Int. 2009;30(7):613-618.

4. Chi TD, Toolan BC, Sangeorzan BJ, Hansen ST. The lateral column lengthening and medial column stabilization procedures. Clin Orthop Rel Res. 1999;365:81-89.

5. DuMontier T, Falicov A, Mosca V, Sangeorzan B. Calcaneal lengthening: investigation of deformity correction in a cadaver flatfoot model. Foot Ankle Int. 2005;26(2):166-170.

6. Evans D. Calcaneo-valgus deformity. J Bone Joint Surg. 1975;57(B):270-278.

7. Flemister AS, Baumhauer JF, Digiovanni BF. Flexor digitorum longus to posterior tibialis transfer with lateral column lengthening for stage II posterior tibial tendon dysfunction. Tech Foot Ankle Surg. 2007;6(1):1-8.

8. Glasoe WM, Grebing BR, Beck S, et al. A comparison of device measures of dorsal first ray mobility. Foot Ankle Int. 2005;26:957-961.

9. Glasoe WM, Yack HJ, Saltzman CL. The reliability and validity of a first ray measurement device. Foot Ankle Int. 2000;21:240-246. 
10. Haeseker G, Mureau MA, Faber FW. Lateral column lengthening for acquired adult flatfoot deformity caused by posterior tibial tendon dysfunction stage II: a retrospective comparison of calcaneus osteotomy with calcaneocuboid distraction arthrodesis. J Foot Ankle Surg. 2010;49(4):380-384.

11. Hiller L, Pinney SJ. Surgical treatment of acquired flatfoot deformity: what is the state of practice among academic foot and ankle surgeons in 2002? Foot Ankle Int. 2003;24:701-705.

12. Hintermann B, Valderrabano V, Kundert HP. Lengthening of the lateral column and reconstruction of the medial soft tissue for treatment of acquired flatfoot deformity associated with insufficiency of the posterior tibial tendon. Foot Ankle Int. 1999;20:622-629.

13. Houck J, Neville C, Tome J, Flemister A. Foot kinematics during a bilateral heel rise test in participants with stage II posterior tibial tendon dysfunction. J Orthop Sports Phys Ther. 2009;39(8):593-603.

14. Houck JR, Tome JM, Nawoczenski DA. Subtalar neutral position as an offset for a kinematic model of the foot during walking. Gait Posture. 2008;28:29-37.

15. Houck JR, Neville CG, Tome J, et al. Ankle and foot kinematics associated with stage II PTTD during stance. Foot Ankle Int. 2009;30:530-539.

16. Hunsaker F, Cioffi D, Amadio P, Wright J, Caughlin B. American Academy of Orthopedic Surgeons outcomes instruments: normative values from the general population. $J$ Bone Joint Surg. 2002;84:208-215.

17. Jan MH, Chai HM, Lin YF, et al. Effects of age and sex on the results of an ankle plantar-flexor manual muscle test. Phys Ther. 2005;85(10):1078-1084.

18. Johnson KA, Strom DE. Tibialis posterior tendon dysfunction. Clin Orthop. 1989;239:196-206.

19. Marks R, Long J, Ness M, Khazzam M, Harris G. Surgical reconstruction of posterior tibial tendon dysfunction: prospective comparison of flexor digitorum longus substitution combined with lateral column lengthening or medial displacement calcaneal osteotomy. Gait Posture. 2009;29:17-22.

20. Martin DP, Engelberg R, Agel J, Swiontkowski MF. Comparison of the Musculoskeletal Function Assessment questionnaire with the Short Form-36, the Western Ontario and McMaster Universities Osteoarthritis Index, and the Sickness
Impact Profile health-status measures. J Bone Joint Surg Am. 1997;79(9):1323-1335.

21. Mosier-LaClair S, Pomeroy G, Manoli A. Intermediate follow-up on the double osteotomy and tendon transfer procedure for stage II posterior tibial tendon insufficiency. Foot Ankle Int. 2001;22(4):283-291.

22. Myerson M. Adult acquired flatfoot deformity: treatment of dysfunction of the posterior tibial tendon. J Bone Joint Surg Am. 1996;78-A:780-792.

23. Neville C, Flemister AS, Houck JR. Deep posterior compartment strength and foot kinematics in subjects with stage II posterior tibial tendon dysfunction. Foot Ankle Int. 2010;31:320-328.

24. Pedowitz WJ, Kovatis P. Flatfoot in the adult. J Am Acad Orthop Surg. 1995;3:293-302.

25. Philbin TM, Pokabla C, Berlet GC. Lateral column lengthening using allograft interposition and cervical plate fixation. Foot Ankle Spec. 2008;1(5):288-298.

26. Sangeorzan BJ, Mosca V, Hansen ST. Effect of calcaneal lengthening on relationships among the hindfoot, midfoot, and forefoot. Foot Ankle. 1993;14:136-141.

27. Swiontkowski MF, Engelberg R, Martin DP, Agel J. Short musculoskeletal function assessment questionnaire: validity, reliability, and responsiveness. J Bone Joint Surg Am. 1999;81(9):1245-1260.

28. Tellisi N, Lobo M, O’Malley M, Kennedy J, Elliott A, Deland J. Functional outcome after surgical reconstruction of posterior tibial tendon insufficiency in patients under 50 years. Foot Ankle Int. 2008;29:1179-1183.

29. Tome J, Nawoczenski DA, Flemister A, Houck J. Comparison of foot kinematics between subjects with posterior tibialis tendon dysfunction and healthy controls. J Orthop Sports Phys Ther. 2006;36:635-644.

30. Umberger BR, Nawoczenski DA, Baumhauer JF. Reliability and validity of first metatarsophalangeal joint orientation measured with an electromagnetic tracking device. Clin Biomech. 1999;14:74-76.

31. Williams DS, McClay IS. Measurements used to characterize the foot and the medial longitudinal arch: reliability and validity. Phys Ther. 2000;80(9):864-871.

32. Younger AS, Sawatzky B, Dryden P. Radiographic assessment of adult flatfoot. Foot Ankle Int. 2005;26:820-825. 